The authors conclude that HCV-infected patients who do not receive interferon treatment develop malignant lymphoma at the rate of $0.23 \%$ per year. Patients who are treated with interferon and who show good clearance of virus are well protected against the development of malignant lymphoma.

Original article Kawamura Y et al. (2007) Viral elimination reduces incidence of malignant lymphoma in patients with hepatitis C. Am J Med 120: 1034-1041

\section{Early complete remission is a survival indicator in only a small subset of multiple myeloma cases}

Some studies have suggested that the achievement of complete remission (CR) in patients with multiple myeloma might be a crucial event associated with superior survival rates, but other trials have failed to demonstrate any link between the two. To investigate these apparently contradictory results, Haessler et al. studied 668 patients with multiple myeloma, following them from diagnosis through treatment with a tandem autotransplant regimen. Time taken to achieve CR was measured and the relationships between CR and event-free survival and overall survival were assessed. The researchers focused on both standard techniques for identifying prognostic factors and the new technique of gene-expression profiling.

Statistical analysis of standard prognostic factors suggested that $C R$ was generally linked with better survival in all patients. Gene-expression profiling, however, highlighted a specific group of $13 \%$ of patients with high-risk disease. In this subgroup, a shorter time to CR was associated with a considerably greater chance of overall survival and event-free survival. CR had no impact on survival in the $87 \%$ of patients who had low-risk disease. It is clear that geneexpression profiling can be used to identify a small subgroup of patients whose long-term survival critically depends on achieving $C R$ at an early stage of treatment. These results indicate that $\mathrm{CR}$ is not generally linked with overall survival or event-free survival, and future clinical trials involving patients with multiple myeloma need to take these findings into account.

Original article Haessler J et al. (2007) Benefit of complete response in multiple myeloma limited to high-risk subgroup identified by gene expression profiling. Clin Cancer Res 13: 7073-7079

\section{Cell-cycle regulators might be useful diagnostic markers in differentiated thyroid cancer}

Patients with differentiated thyroid cancer (DTC) have a favorable prognosis; however, in some cases patients succumb to their disease because of local recurrence and/or distant metastases. There is a need to identify markers that can predict tumor behavior. A study by Melck et al. has assessed the diagnostic and prognostic utility of cell-cycle markers in DTC that have previously been implicated in other malignancies.

The expression profiles of the cell-cycle regulators p16, p21, p27, p53, p57, p63, cyclin D1, cyclin $\mathrm{E}$ and $\mathrm{mdm} 2$ were analyzed with use of tissue microarrays that contained samples from 100 benign and 105 malignant lesions and 24 lymph nodes. Compared with benign lesions, DTCs had significantly higher expression of p16 (5\% vs $54.7 \%)$, p21 (38\% vs $71.7 \%)$, cyclin D1 $(45.7 \%$ vs $87.1 \%)$ and cyclin E (37.4\% vs $72.3 \% ; P<0.001$ for all comparisons). Both malignant and benign samples showed either low-level or no staining for p57 and p63, and there were no significant differences in the expression of p27, p53, and mdm2 between the benign and malignant samples. Positive p16 staining was noted in $73 \%$ of samples from patients with lymph-node metastases, whereas only $45 \%$ of samples from patients with no lymph-node involvement stained positive for p16 $(P=0.01)$. Moreover, p16 immunoreactivity significantly correlated with the presence of lymph-node metastases $(P=0.03)$ and extrathyroidal tumor extension $(P=0.02)$. An inverse correlation between $\mathrm{p} 27$ expression and tumor multifocality was observed $(P=0.011)$.

This study suggests that cell-cycle regulators might be useful markers for the diagnosis and prognosis of DTC.

Original article Melck A et al. (2007) Cell cycle regulators show diagnostic and prognostic utility for differentiated thyroid cancer. Ann Surg Oncol 14: 3403-3411

\section{Panitumumab confers QoL benefits on patients with metastatic colorectal cancer}

Panitumumab, the first fully human antibody that targets the EGFR, significantly improved the progression-free survival of patients with metastatic colorectal cancer (CRC) in a phase III 